\title{
Запрещенное резонансное комбинационное рассеяние света в сверхрешетках GaAs/AIAs: эксперимент и расчеты
}

\author{
(C) В.А. Володин ${ }^{1,2}$, В.А. Сачков ${ }^{3}$, М.П. Синюков ${ }^{1}$ \\ ${ }^{1}$ Институт ффизики полупроводников им. А.В. Ржанова Сибирского отделения Российской академии наук, \\ 630090 Новосибирск, Россия \\ ${ }^{2}$ Новосибирский государственный университет, \\ 630090 Новосибирск, Россия \\ ${ }^{3}$ Омский научный центр Сибирского отделения Российской академии наук, \\ 644024 Омск, Россия \\ E-mail: volodin@isp.nsc.ru
}

(Получена 20 июня 2017 г. Принята к печати 26 июня 2017 г.)

Рассчитаны и экспериментально исследованы спектры комбинационного рассеяния света сверхрешеток $\mathrm{GaAs} / \mathrm{AlAs}(001)$ для различных направлений волнового вектора. Эксперименты проводились с применением конфокального оптического микроскопа, совмещенного со спектрометром комбинационного рассеяния света (micro-Raman) в различных геометриях рассеяния, как для фононов с волновым вектором, направленным по нормали к сверхрешетке, так и вдоль ее слоев (в англоязычной литературе „in-plane“ геометрия). Частоты и собственные векторы фононов были рассчитаны в приближении расширенной модели Борна с учетом кулоновского взаимодействия в приближении жестких ионов. Спектры комбинационного рассеяния света были рассчитаны в рамках механизма деформационного потенциала; при этом оказалось, что в экспериментальных спектрах проявляются дополнительные пики, не описывающиеся в рамках данного подхода. Возможно, эти пики возникают вследствие проявления в резонансных условиях запрещенного правилами отбора комбинационного рассеяния света. Сделана попытка объяснить появление данных пиков в экспериментальных спектрах в рамках неупругого рассеяния фотонов на связанных зарядах (фононах с большим дипольным моментом).

DOI: 10.21883/FTP.2018.06.45917.8671

\section{1. Введение}

Сверхрешетки это гетероструктуры с искусственно заданным периодом, который определяет их зонную структуру, оптические и колебательные свойства [1-3]. Наиболее заметные эффекты фононного спектра сверхрешеток - это „свертка“ акустических и локализация оптических мод [4-10]. С развитием техники применения оптической микроскопии в комбинационном рассеянии света (micro-Raman) появилась возможность исследовать зависимость дисперсии оптических фононов в сверхрешетках в зависимости от направления волнового вектора, в том числе и в геометрии, когда волновой вектор лежит в плоскости сверхрешетки (,in-plane“ геометрия) [11-22].

Резонансные явления в комбинационном рассеянии света привлекают внимание исследователей уже почти 50 лет [23,24]. Исходя из распространенного подхода диаграмм Фейнмана один из возможных механизмов для стоксова процесса выглядит так: фотон рождает $N$ виртуальных фононов, затем $N-1$ из этих фононов рождают рассеянный фотон, а один из фононов становится реальным. Но, так как энергия фотона видимого диапазона в 20-30 раз превышает энергию оптических фононов, вероятность комбинационного рассеяния света по механизму прямого взаимодействия невелика [23], поэтому процесс неупругого рассеяния фотона на фононах идет посредством взаимодействия с электронной подсистемой кристалла. В одной из последовательностей процессов диаграмм Фейнмана механизм для стоксова процесса выглядит так: фотон рождает виртуальную электрон-дырочную пару; электрон (либо дырка) рождает фонон, потом электрон-дырочная пара рекомбинирует с рождением рассеянного фотона. Рассчитать вероятность электрон-фононного взаимодействия из первых принципов - это весьма сложная задача, поэтому разделяют несколько феноменологических механизмов. Механизм деформационного потенциала (будем далее обозначать его DP - deformation potential) можно учесть в подходе поляризуемости связей Волькенштейна, в котором поляризуемость валентных связей в молекулах или кристаллах модифицирована смещением атомов, а значит, поляризуемость среды колеблется с частотой фононов [24-26]. Электрооптический механизм основан на модуляции поляризуемости связей макроскопическими полями, индуцированными смещениями катионов и анионов $[24,27,28]$. Зачастую из электрооптического механизма выделяют так называемый фрёлиховский (Fröhlich) механизм [24,29]. Это нелокальное, кулоновское взаимодействие электрона (или дырки) с фононом. Известно, что данный механизм проявляется в резонансных условиях рассеяния [23,24] и нарушает правила отбора, характерные для DP. Учесть вклад этого механизма в спектры комбинационного рассеяния света из первых принципов очень сложная задача. Данная работа - это попытка экспериментально исследовать вклад запрещенного в рамках DP и электрооптического механизмов рассеяния, а также учесть его в модельных 
спектрах, используя простые феноменологические подходы в рамках классической электродинамики.

\section{2. Эксперимент}

Сверхрешетки GaAs/AlAs были выращены с применением метода молекулярно-лучевой эпитаксии на подложке GaAs c ориентацией (001). Сначала выращивались толстые буферные слои GaAs и AlAs, затем чередующиеся слои AlAs и GaAs. Исследуемая структура содержала 103 периода по 7 монослоев (MC) AlAs и $\mathrm{GaAs}$, общая толщина сверхрешетки составила 412 нм. Для регистрации спектров использовался спектрометр комбинационного рассеяния света T64000 (Horiba Jobin Yvon) с конфокальным микроскопом Olympus с короткофокусным стократным объективом (отношение апертуры к фокусному расстоянию $1 / 2.5$ ), для возбуждения использовалась линия 660 нм твердотельного лазера. Спектральное разрешение было не хуже $1.5 \mathrm{~cm}^{-1}$. Мощность лазерного излучения, достигающая образца, составляла менее 1 мВт, излучение не приводило к заметному нагреву образцов. Пространственное разрешение определялось размерами пучка в фокусе и составляло 1 микрометр. Это позволило освещать сверхрешетку с „торца“ и применять геометрию, в которой волновые векторы падающего и рассеянного фотонов лежат в плоскости сверхрешетки (так называемая ,in-plane“ геометрия $[12,14])$. Данный подход описан более детально в работах $[17,18,28]$.

\section{3. Результаты и обсуждение}

Как уже отмечалось, запрещенные в обычных условиях механизмы комбинационного рассеяния света могут проявляться в резонансных условиях [24]. Согласно расчетным и экспериментальным данным [30], энергия для прямых оптических переходов в сверхрешетке $\mathrm{GaAs}_{7} / \mathrm{AlAs}_{7}$ составляет 1.91 эВ. На рис. 1 показан спектр фотолюминесценции исследуемой сверхрешетки. Видны широкие полосы сигналов в красном диапазоне (по-видимому, от прямых переходов) и на границе инфракрасного диапазона. В случае такой короткопериодной сверхрешетки энергии прямых оптических переходов выше, чем непрямых [31]. Вероятно, полоса фотолюминесценции на границе инфракрасного диапазона обусловлена непрямыми переходами. Для исследования комбинационного рассеяния света в резонансных условиях (вблизи 1.91 эВ) был выбран твердотельный лазер с длиной волны 660 нм. Энергия фотона (1.89 эВ) в данном случае находится вблизи максимума сигнала фотолюминесценции. Стоит отметить, что сверхрешетки с другим количеством монослоев (исследованные в работах $[18,28,32])$ обладали таким большим сигналом фотолюминесценции при возбуждении данным лазером, что невозможно было на таком большом фоне сигнала фотолюминесценции исследовать вклад от комбинационного рассеяния света.
Как видно из рис. 2 („нормальная“ геометрия рассеяния, волновые векторы перпендикулярны плоскости сверхрешетки), в спектре присутствует сильный фон от

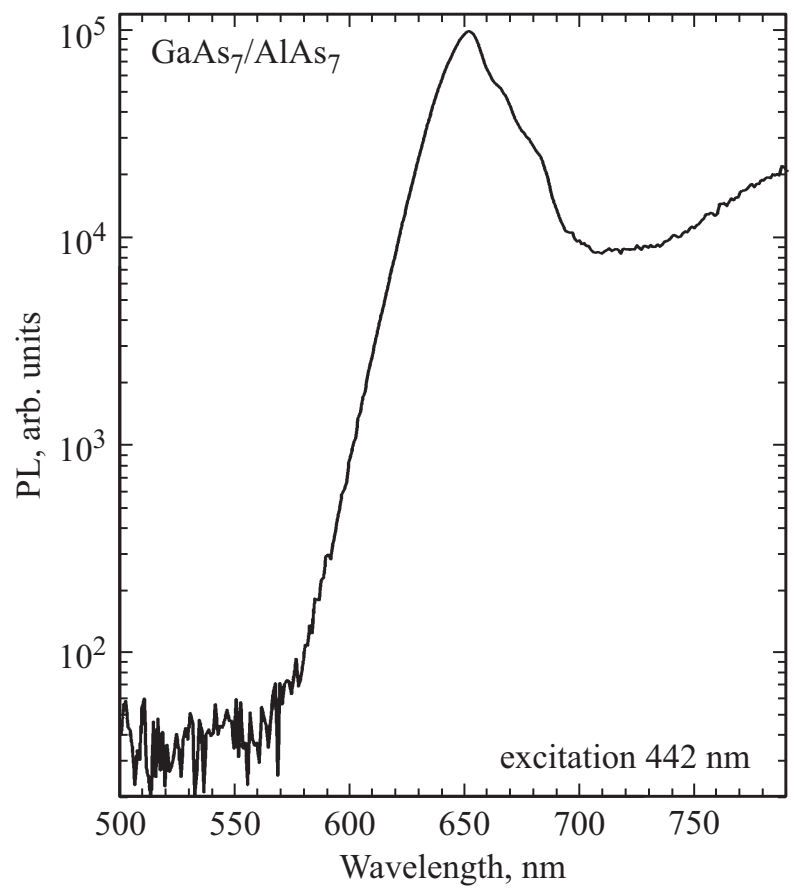

Рис. 1. Спектр фотолюминесценции сверхрешетки $\mathrm{GaAs}_{7} / \mathrm{AlAs}_{7}$ при возбуждении фотонами с энергией 2.81 эВ.

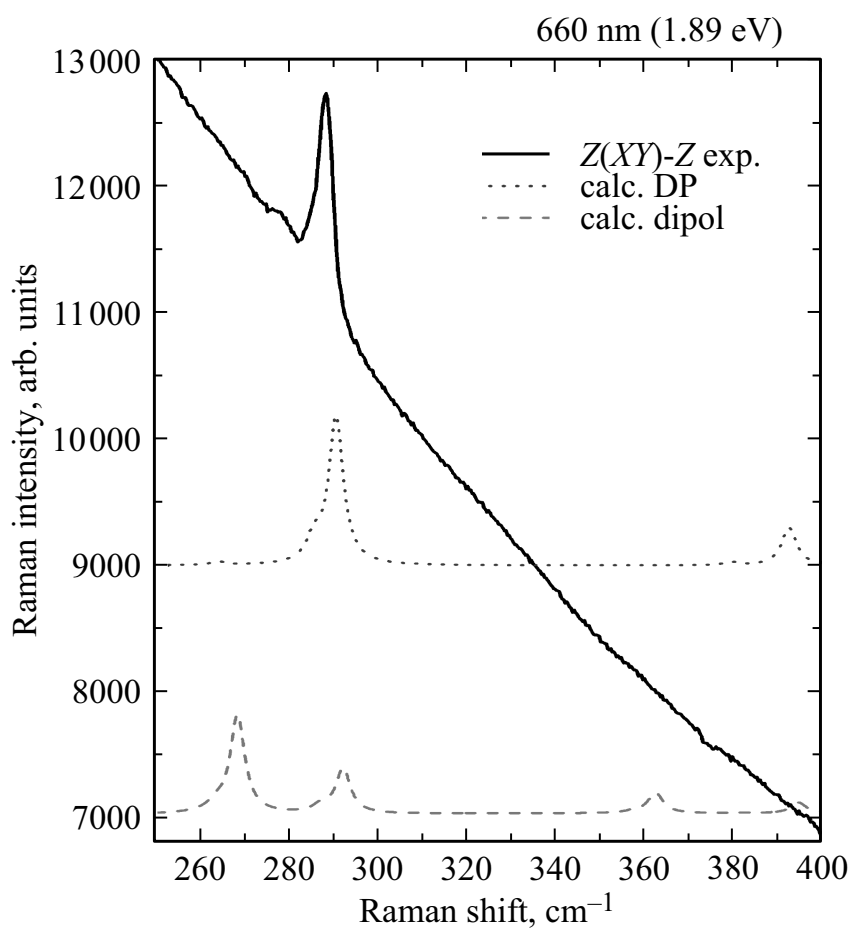

Рис. 2. Экспериментальные (сплошная кривая) и рассчитанные (точечная кривая - модель деформационного потенциала, штриховая кривая - модель дипольного взаимодействия) спектры комбинационного рассеяния света сверхрешетки $\mathrm{GaAs}_{7} / \mathrm{AlAs}_{7}$. „Нормальная““ геометрия рассеяния $Z(X Y)-Z$. 
фотолюминесценции, что не позволяет исследовать пики комбинационного рассеяния света малой интенсивности. Геометрия рассеяния в данном случае $Z(X Y)-Z$. Здесь и далее оси в скобках соответствуют направлению поляризации вектора напряженности электрического поля падающей и рассеянной электромагнитных волн, а оси вне скобок показывают направление волнового вектора падающей и рассеянной электромагнитных волн - так называемые обозначения Порто [24]. Оси $X, Y$ и $Z$ параллельны кристаллографическим направлениям (100), (010) и (001) соответственно. В спектре отчетливо виден только наиболее интенсивный пик при $288 \mathrm{~cm}^{-1}$, от рассеяния на продольных оптических (LO) фононах, локализованных в слоях GaAs. Это пик именно от комбинационного рассеяния, а не особенность фотолюминесценции, так как его ширина мала, к тому же он проявлялся с тем же сдвигом в $288 \mathrm{~cm}^{-1}$ и при возбуждении лазером с другой длиной волны (633 нм). На рис. 2 представлены также рассчитанные спектры для различных механизмов рассеяния. Детали расчетов будут обсуждены позже. Отметим, что модель деформационного потенциала удовлетворительно описывает экспериментальный спектр.

На рис. 3 представлены спектры для геометрии рассеяния, в которой волновые векторы падающего и рассеянного света лежат в плоскости сверхрешетки, вдоль направления $X^{\prime}$. Направления осей $X^{\prime}$ и $Y^{\prime}-$ это кристаллографические направления (110) и (110) соответственно. Вверху и внизу приведены спектры, полученные в геометриях $X^{\prime}\left(Y^{\prime} Y^{\prime}\right)-X^{\prime}$ и $X^{\prime}\left(Z Y^{\prime}\right)-X^{\prime}$. В обеих поляризациях в экспериментальном спектре присутствует пик с положением $275 \mathrm{~cm}^{-1}$. Данная мода - это разрешенный фонон $\mathrm{TO}_{z}+\mathrm{IF}[18]$, пик от него виден в рассчитанных спектрах, но положение его составляет $278 \mathrm{~cm}^{-1}$. Как было показано в работе [18], сдвиг в $3 \mathrm{~cm}^{-1}$ обусловлен перемешиванием атомов галлия и алюминия на гетерогранице в реальной сверхрешетке. В экспериментальном спектре в геометрии $X^{\prime}\left(Y^{\prime} Y^{\prime}\right)-X^{\prime}$ присутствует также низкочастотное плечо, которое обусловлено рассеянием на $\mathrm{TO}_{z}+$ IF-модах более высокого порядка, они также присутствуют в расчетах. Широкие особенности в экспериментальных спектрах в области частот оптических колебаний связей алюминий-мышьяк также соответствуют $\mathrm{TO}_{z}+\mathrm{IF}$-модам в слоях AlAs. В экспериментальных спектрах они сильно уширены и „размыты“ по сравнению с рассчитанными спектрами, очевидно, вследствие перемешивания атомов галлия и алюминия на гетерогранице в реальной сверхрешетке [18].

Обратимся к геометрии $X^{\prime}\left(Z Y^{\prime}\right)-X^{\prime}-$ нижняя часть рис. 3. В данной геометрии в экспериментальном спектре пик с частотой $265 \mathrm{~cm}^{-1}$ больше, чем пик с частотой $275 \mathrm{~cm}^{-1}$. Отметим, что в спектре, рассчитанном с учетом механизма деформационного потенциала, данный высокочастотный пик вообще отсутствует. В расчетах с учетом „дипольного“ взаимодействия (подробности будут обсуждены далее) этот пик присутствует. Учет

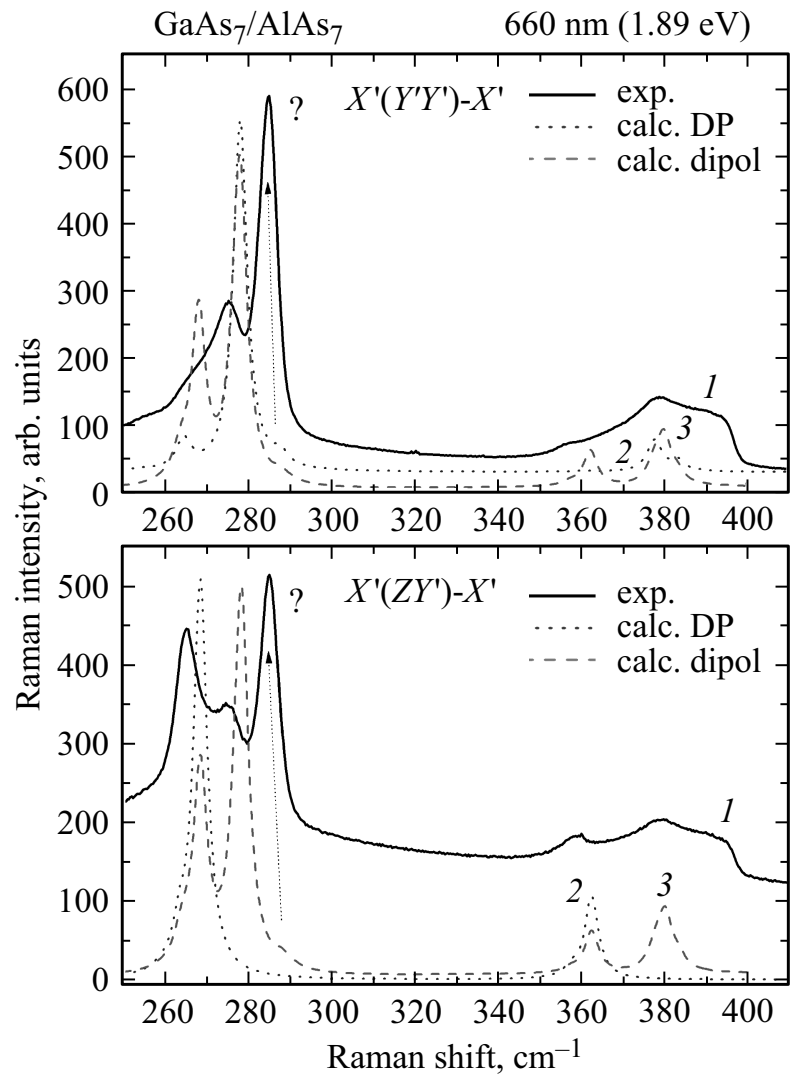

Рис. 3. Экспериментальные (сплошная кривая 1) и рассчитанные (точечная кривая 2 - модель деформационного потенциала, штриховая кривая 3 - модель дипольного взаимодействия) спектры комбинационного рассеяния света сверхрешетки $\mathrm{GaAs}_{7} / \mathrm{AlAs}_{7}$. Геометрия „,in-plain“, вверху $X^{\prime}\left(Y^{\prime} Y^{\prime}\right)-X^{\prime}$, внизу $X^{\prime}\left(Z Y^{\prime}\right)-X^{\prime}$.

обоих механизмов рассеяния хорошо соответствует эксперименту, за исключением пика с частотой $285 \mathrm{~cm}^{-1}$. Видимый в эксперименте в обеих поляризациях, в расчетах по обоим механизмам он присутствует только в виде слабой особенности.

На рис. 4 показаны экспериментальные и рассчитанные спектры в геометриях $X(Z Y)-X$ и $X(Y Y)-X$. В экспериментальных спектрах в обеих поляризациях присутствуют три пика с положениями 285, 275 и $265 \mathrm{~cm}^{-1}$. В различных поляризациях интенсивности данных пиков различны, так пик с положением $265 \mathrm{~cm}^{-1}$ в поляризации $(Z Y)$ максимален, а в поляризации $(Y Y)$, напротив, минимален и проявляется только в виде слабого плеча. В области частот оптических колебаний Al-As также присутствуют три широких пика, самый интенсивный имеет положение $380 \mathrm{~cm}^{-1}$. Отметим, что расчеты в приближении деформационного потенциала дают только 2 интенсивных пика $278 \mathrm{~cm}^{-1}\left(\mathrm{LO}_{x}+\mathrm{IF}-\right.$ мода в слоях $\left.\mathrm{GaAs}\right)$ и $380 \mathrm{~cm}^{-1}$ $\left(\mathrm{LO}_{x}+\mathrm{IF}-\right.$ мода в слоях AlAs) только в поляризации $(Z Y)$. В рассчитанных спектрах с учетом, „дипольного“ взаимодействия проявляются также дополнительные пики с частотами 268 и $362 \mathrm{~cm}^{-1}$. Экспериментально 


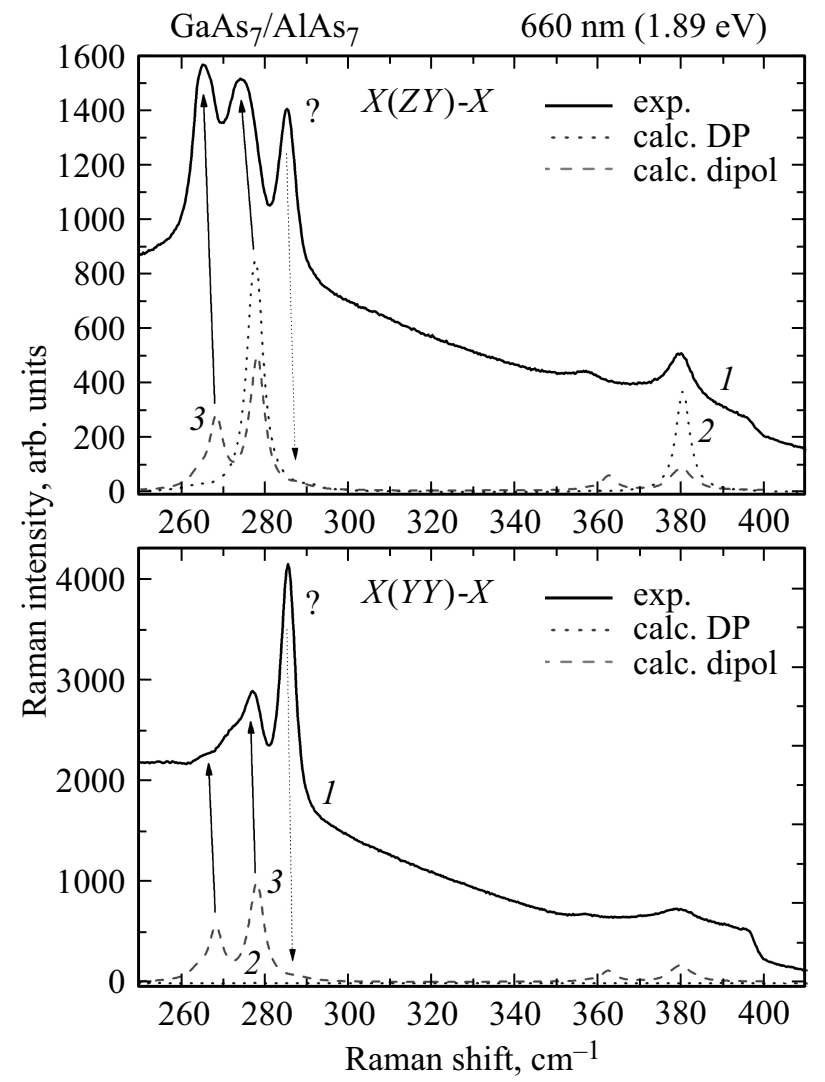

Рис. 4. Экспериментальные (сплошная кривая 1) и рассчитанные (точечная кривая 2 - модель деформационного потенциала, штриховая кривая 3 - модель дипольного взаимодействия) спектры комбинационного рассеяния света сверхрешетки $\mathrm{GaAs}_{7} / \mathrm{AlAs}$ 7. Геометрия „in-plain“, вверху $X(Z Y)-X$, внизу $X(Y Y)-X$.

наблюдаемый пик с частотой $285 \mathrm{~cm}^{-1}$ не активен как в DP, так и в ,дипольном“ механизмах.

Обратимся теперь к деталям расчетов. Для расчетов фононного спектра была использована феноменологическая модель Борна (приближение двухчастичного взаимодействия) [33], с учетом не только ближайших соседей, но и следующих координационных сфер (так называемая расширенная модель Борна). Для моделирования фононов в гетероструктурах GaAs/AlAs использовался метод массо-замещения [26] на основе модельных параметров арсенида галлия (в случае атома алюминия менялась только масса). Учет дальнодействующего кулоновского взаимодействия проводился в дипольном приближении. Эффективный заряд катионов и анионов был найден из разницы квадратов продольной $\left(\omega_{L}=292.6 \mathrm{~cm}^{-1}\right)$ и поперечной $\left(\omega_{T}=269 \mathrm{~cm}^{-1}\right)$ частот. Интенсивность пика для каждой моды вычислялась в приближении аддитивной поляризуемости связей Волькенштейна $[25,26]$. Этот метод основывается на предположении, что каждая ковалентная связь имеет свою поляризуемость, являющуюся функцией только длины этой связи. Тогда поляризуемость сверхрешетки можно представить как сумму поляризуемостей всех связей. Ис- пользовались параметры поляризуемости из работы [34]. Более детально расчеты описаны в работах $[18,26]$.

Спектры, показанные на рис. 2-4 точечными кривыми, были рассчитаны с учетом только механизма деформационного потенциала. Как уже отмечалось, они не обнаруживают всех экспериментальных пиков. Спектры, рассчитанные с учетом электрооптического механизма рассеяния, не слишком отличались от спектров, рассчитанных для деформационного потенциала. Это связано с тем, что для макроскопического электрического поля (которое практически не меняется на протяжении примитивной ячейки - случай длинноволновых фононов), если сложить вклады от всех связей кристалла с ячейкой типа цинковой обманки, результирующие тензоры будут иметь практически такой же вид, как и в случае механизма деформационного потенциала [28]. Вклад от перемешивания атомов на гетерограницах GaAs/AlAs в спектры комбинационного рассеяния света был также оценен и рассчитан, как в работе [18]. Было установлено, что даже полное перемешивание либо наличие не плоской (корругированной) гетерограницы не ведут к появлению в спектрах интенсивных мод с частотами 265 и $285 \mathrm{~cm}^{-1}$ (такие моды видны в экспериментальных спектрах). Таким образом, разупорядочение не может быть причиной сильного нарушения правил отбора. Были сделаны оценки того, как „утечка поляризации“ изза используемого светосильного объектива микроскопа может нарушить правила отбора. Поскольку показатель преломления сверхрешеток большой (около 4), отношение параллельной компоненты волнового числа к перпендикулярной компоненте для квазиобратного рассеяния в нашем случае составляет около $1 / 10$. Поскольку интенсивность рассеянного света пропорциональна квадрату амплитуды, вклад эффекта утечки поляризации не превышает 1\% [28].

Примитивная ячейка сверхрешетки $\mathrm{GaAs}_{7} / \mathrm{AlAs}_{7}$ coдержит 28 атомов. Таким образом, существуют 84 фононных моды, половина из которых относится к локализованным оптическим ветвям. Мы рассчитали собственные частоты и собственные векторы, а также электрический дипольный момент этих 84 мод для волнового вектора $10^{6} \mathrm{~cm}^{-1}$ вдоль осей $Z, X^{\prime}$ и $X$. В работе [28] было предположено, что моды с большими дипольными моментами могут взаимодействовать с заряженными частицами (электронами или дырками) в сверхрешетках за счет кулоновского взаимодействия. В более общем случае кулоновский механизм электрон-фононного взаимодействия известен как фрёлиховский механизм, проявляющийся в транспортных явлениях, а также в комбинационном рассеянии света [3,24-31]. Интенсивность фрёлиховского рассеяния обычно пропорциональна квадрату волнового вектора, его часто связывают с квадрупольным механизмом [24]. Мы предлагаем кулоновский механизм рассеяния, но дипольный.

Известна „квазиклассическая“ интерпретация комбинационного рассеяния света как аналог релеевского рассеяния на связанном заряде с учетом ангармонизма 
колебаний $[35,36]$. В этом случае амплитуда рассеянной волны также пропорциональна ангармоническому вкладу и амплитуде колебаний (т.е. дипольному моменту). Интенсивность рассеянной волны, таким образом, будет пропорциональна квадрату амплитуды дипольного момента. В таком подходе правила отбора не зависят от поляризации, а зависят только от направления волнового вектора фонона (так как от него зависит дипольный момент). Таким образом, если фононная мода обладает электрическим дипольным моментом, она создает возмущающее электрическое поле для свободных электронов и дырок, и в целом, поляризуемость системы промодулирована по времени с частотой данной моды, так как такой механизм является кулоновским и не локален. Если предположить, что интенсивность такого рассеяния пропорциональна квадрату модуля дипольного момента, то можно рассчитать спектр комбинационного рассеяния света для всех направлений волнового вектора. Именно это и было сделано, а расчеты представлены на рис. 2-4 штриховыми кривыми.

Как уже было отмечено выше, в экспериментальных спектрах присутствует интенсивная мода с частотой $285 \mathrm{~cm}^{-1}$, а в рассчитанных с учетом „дипольного“ взаимодействия спектрах она не обладает максимальным дипольным моментом, а присутствует в виде относительно слабого пика. В расчетах присутствует мода с частотой $288 \mathrm{~cm}^{-1}$, распространяющаяся как вдоль оси $X^{\prime}$, так и вдоль оси $X$, однако ее дипольный момент (направленный в основном вдоль оси $Z$ ) в $\sim 4.5$ раза меньше по амплитуде, чем дипольный момент моды с частотой $278 \mathrm{~cm}^{-1}$. Таким образом, ее интенсивность почти в 20 раз меньше, как видно из рис. 3 и 4. Такое отличие от эксперимента требует дополнительных исследований. Возможно, что ангармонизм данной высокочастотной моды больше, чем у моды с частотой $278 \mathrm{~cm}^{-1}$. Возможно, эта мода проявляется вследствие фрёлиховского механизма рассеяния. Однако, как отмечено в [24], фрёлиховский механизм проявляется только в параллельной геометрии рассеяния по поляризации, в эксперименте же эта мода проявляется в скрещенной поляризации даже более заметно (рис. 3, нижняя часть и рис. 4 , верхняя часть). Возможны также нарушения правил отбора, связанные с дефектами на гетерограницах, либо со встроенными электрическими полями в сверхрешетке. Известно, что постоянное электрическое поле меняет симметрию сверхрешеток и может нарушать правила отбора [37]. Нарушение правил отбора в сверхрешетке с толщиной 412 нм может быть связано с тем, что этот слой фактически является волноводом (нижней границей которого является подложка GaAs, а верхней границей - воздух), в котором свет затухает и рассеивается, меняя свое направление, поэтому правила отбора смягчаются. Тем не менее предложенный учет механизма рассеяния в рамках классической электродинамики позволяет более адекватно моделировать спектры комбинационного рассеяния в условиях, близких к резонансному рассеянию.

\section{4. Заключение}

Таким образом, в работе обнаружено различие между рассчитанными (с учетом деформационного механизма) и экспериментальными спектрами комбинационного рассеяния света сверхрешеток GaAs/AlAs, зарегистрированными в резонансных условиях рассеяния в ,іnplain“ геометрии. Предложен простой подход для учета в рассеянии запрещенных деформационным и электрооптическим механизмами фононных мод, основанный на „Дипольном“ приближении.

Работа выполнена по государственному заданию Программа фундаментальных исследований СО РАН № II.9.4.

\section{Список литературы}

[1] Ж.И. Алфёров. ФТП, 32, 3 (1998).

[2] А.П. Силин. Успехи физ. наук, 147, 485 (1985).

[3] Light scattering in Solids V. Superlattices and other microstructures / ed. by M. Cardona, G. Guntherodt. (Berlin, Springer Verlag, 1989).

[4] С.М. Рытов. ЖЭТФ, 29, 605 (1955).

[5] A.S. Barker, jr., J.L. Merz, A.C. Gossard. Phys. Rev. B, 17, 3181 (1978).

[6] R. Merlin, C. Colvard, M.V. Klein, H. Morkoc, A.Y. Cho, A.C. Gossard. Appl. Phys. Lett., 36, 43 (1980).

[7] B. Jusserand, D. Paquet, J. Kervarec, A. Regreny. J. Phys., 45, C5 (1984).

[8] A.K. Sood, J. Menendez, M. Cardona, K. Ploog. Phys. Rev. Lett., 54, 2115 (1985).

[9] M. Cardona. Superlat. Microstr., 5, 27 (1989).

[10] V.A. Volodin, M.D. Efremov, V.V. Preobrazhenskii, B.R. Semyagin, V.V. Bolotov, Superlat. Microstr., 26, 11 (1999).

[11] A. Huber, T. Egeler, W. Ettmiiller, H. Rothfritz, G. Trankle, G. Abstreiter. Superlat. Microstr., 9, 309 (1991).

[12] R. Hessmer, A. Huber, T. Egeler, M. Haines, G. Trankle, G. Weimann, G. Abstreiter. Phys. Rev. B, 46, 4071 (1992).

[13] G. Scamarcio, M. Haines, G. Abstreiter, E. Molinari, S. Baroni, A. Fischer, K. Ploog. Phys. Rev. B, 47, 1483 (1993).

[14] A. Fainstein, P. Etchegoin, M.P. Chamberlain, M. Cardona, K. Totemeyer, K. Eberl. Phys. Rev. B, 51, 14448 (1995).

[15] M. Zunke, R. Schorer, G. Abstreiter, W. Klein, G. Weimann, M.P. Chamberlain. Sol. St. Commun., 93, 847 (1995).

[16] V.A. Volodin, M.P. Sinyukov, V.A. Sachkov, M. Stoffel, H. Rinnert, M. Vergnat. Europhys. Lett., 105, 16003 (2014).

[17] В.А. Володин, М.П. Синюков. Письма ЖЭТФ, 99, 463 (2014).

[18] В.А. Володин, В.А. Сачков, М.П. Синюков. ЖЭТФ, 147, 906 (2015).

[19] V.A. Volodin, V.A. Sachkov, I.S. Golovin. Physics Express, 4, 11 (2014).

[20] Shang-Fen Ren, Hanyou Chu, Yia-Chung Chang. Phys. Rev. B, 37, 8899 (1988).

[21] Shang-Fen Ren, G. Qin. Sol. St. Commun., 121, 171 (2002).

[22] M.P. Chamberlain, M. Cardona, B.K. Ridley. Phys. Rev. B, 48, 14356 (1993).

[23] R.M. Martin. Phys Rev. B, 4, 3676 (1971).

[24] Рассеяние света в твердых телах: проблемы прикладной физики, под ред. М. Кардоны (М., Мир, 1979). 
[25] М.В. Волькенштейн. Докл. АН СССР, XXXII, 185 (1941).

[26] В.А. Сачков. Автореф. канд. дис. (Омск, 2011).

[27] L.N. Ovander, N.S. Tyu. Phys. Status Solidi B, 91, 763 (1979).

[28] V.A. Volodin, V.A. Sachkov, M.P. Sinyukov. ЖЭТФ, 150, 184 (2016).

[29] H. Fröhlich. Adv. Phys., 3, 325 (1954).

[30] H. Fujimoto, C. Hamaguchi, T. Nakazava, K. Taniguchi, K. Imanichi, H. Kato, Y. Watanabe. Phys. Rev. B, 41, 7593 (1990).

[31] A.W.E. Minnaert, A.Yu. Silov, W. van der Vleuten, J.E.M. Haverkoff, J.H. Wolter. Phys. Rev. B, 63, 075303 (2001).

[32] V.A. Volodin, G.K. Krivyakin, V.A. Sachkov, M.P. Sinyukov. Proc. 24th Intern. Symp. „Nanostructures: Physics and Technology“ (June 27-July 1, 2016, St. Petersburg) p. 274.

[33] М. Борн, Х. Кунь. Динамическая теория кристаллических решеток (М., ИЛ, 1958).

[34] P. Castrillo, L. Colombo, G. Armelles. Phys. Rev. B, 49, 10362 (1994).

[35] A.S. Barker, jr., R. Loudon. Rev. Mod. Phys., 44, 18 (1972).

[36] И.Н. Мешков, Б.В. Чириков. Электромагнитное поле. Ч. 2: Электромагнитные волны и оптика (Новосибирск, Наука, 1987).

[37] В.А. Гайслер. Автореф. докт. дис. (Новосибирск, 1996).

Редактор Г.А. Оганесян

\title{
Forbidden resonant Raman scattering in GaAs/AIAs superlattices: experiments and calculations
}

\author{
V.A. Volodin ${ }^{1,2}$, V.A. Sachkov ${ }^{3}$, M.P. Sinyukov ${ }^{1}$ \\ ${ }^{1}$ Rzhanov Institute of Semiconductor Physics, \\ Siberian Division of Russian Academy of Sciences, \\ 630090 Novosibirsk, Russia \\ 2 Novosibirsk State University, \\ 630090 Novosibirsk, Russia \\ ${ }^{3}$ Omsk Scientific Center of Siberian Branch, \\ Russian Academy of Sciences, \\ 644024 Omsk, Russia
}

\footnotetext{
Abstract The Raman spectra of GaAs/AlAs(001) superlattices for different directions of the wave vectors are calculated and experimentally studied. The experiments were carried out using micro-Raman setup in various scattering geometries, both for phonons with a wave vector directed along the normal to the superlattice, and along its layers (so-called ,in-plane“ geometry). The frequencies and eigenvectors of phonons were calculated in the approximation of the extended Born model with taking into account Coulomb interaction in the rigid-ion approximation. Raman spectra were calculated within the framework of the deformation potential mechanism; it turned out that in the experimental spectra additional peaks appear that are not described in the framework of this approach. Perhaps these peaks arise due to the manifestation in resonance conditions of the Raman scattering that is forbidden by the selection rules. An attempt was made to explain the appearance of these peaks in experimental spectra in the framework of inelastic scattering of photons by bound charges (phonons with a large dipole moment).
} 